\title{
Analyse de la motilité et du mouvement flagellaire des spermatozoïdes de verrat au cours du transit épididymaire
}

\author{
Kirsten BORK $\left({ }^{1}\right)$, C. CHEVRIER $\left({ }^{2,}{ }^{3}\right)$, M. PAQUIGNON $\left({ }^{2,}{ }^{4}\right)$, P. JOUAN- \\ $\operatorname{NET}\left({ }^{1}\right)$, J. L. DACHEUX $\left({ }^{2,3}\right)\left({ }^{\prime}\right)$ \\ (1) Laboratoire d'Histologie-Embryologie, \\ Centre Hospitalier Universitaire, 94270 Kremlin Bicêtre, France. \\ ( ${ }^{2}$ ) Laboratoire de Physiologie de la Reproduction. I.N.R.A., \\ Nouzilly 37380 Monnaie, France. \\ ${ }^{(3)}$ Laboratoire de Physiologie Comparée, \\ Faculté des Sciences, Parc Grandmont, 37200 Tours, France. \\ $\left({ }^{4}\right)$ Institut Technique du Porc. 149, rue de Bercy, 75595 Paris Cedex 12, France.
}

Summary. Flagellar motility and movement of boar spermatozoa during epididymal transit.

The motility of boar spermatozoa during epididymal transit was analysed in vitro using various techniques. From the head to the cauda there was an increase of the percentage of motile and progressive spermatozoa. During maturation there was a progressive reduction of flagellar bend curvature while flagellar beat frequencies increased. A three dimensional pattern of flagellar beating responsible for cell rotation and straight line progression of spermatozoa was observed only in caudal epididymis. The addition of epididymal fluid protein to the media could increase the number of motile cells at the various levels but had no influence on the characteristics of flagellar bending.

\section{Introduction.}

Les spermatozoïdes de l'ensemble des mammifères subissent en dehors des gonades des transformations indispensables à l'acquisition du pouvoir fécondant. Les gamètes continuent à se différencier dans la lumière du tubule épididymaire. De très nombreux changements soient biochimiques ou soient structurels (en particulier membranaires) (Bedford, 1975 ; Orgebin-Crist et al., 1975) ont déjà été décrits mais les modifications les plus visibles sont les changements concernant leur motilité. En effet, pour de très nombreuses espèces, la motilité des spermatozoïdes, inexistante à la sortie du testicule et dans les premières régions épididymaires, apparaît dans la région antérieure du corps de l'épididyme et est 
totalement acquise pour les gamètes de la région postérieure de cet organe. L'évolution du mouvement du flagelle et la localisation précise de ces modifications dans l'épididyme sont variables entre espèces (Gaddum, 1968). Les conditions d'études de la motilité sont également susceptibles d'influencer et de modifier les caractéristiques de ce paramètre de maturation.

Dans la présente étude, l'évolution du mouvement flagellaire des spermatozoïdes de verrat a été analysée dans les quatre zones épididymaires où les spermatozoïdes subissent des modifications membranaires les plus importantes (Dacheux et al., 1985). L'analyse quantitative et qualitative du mouvement des spermatozoïdes a été effectuée à l'aide de plusieurs techniques et en fonction de différents milieux d'incubation.

Les résultats de cette analyse précisent l'influence des conditions expérimentales sur le profil d'évolution de la motilité des spermatozoïdes dans l'épididyme et caractérisent les différentes étapes du mouvement flagellaire des spermatozoïdes de verrat.

\section{Matériel et techniques.}

\section{Animaux, milieux et conditions d'incubation.}

Le travail a été effectué sur 5 verrats adultes de race Large White. Les spermatozoïdes recueillis à 4 niveaux épididymaires: région 4 (tête distale), région 5 (corps proximal), région 7 (corps distal) et région 9 (queue postérieure) ont été prélevés soit après section des tubules épididymaires dans les régions correspondantes soit par microperfusion du contenu de la lumière du tubule en présence de milieu KRB (Imai et al., 1977). Les gamètes ont ensuite été dilués à

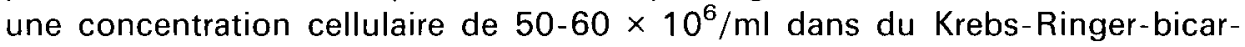
bonate (KRB) contenant ou non $5 \mathrm{mg} / \mathrm{ml}$ d'albumine sérique bovine (BSA) ou des protéines épididymaires présentes dans le liquide de la région postérieure de cet organe et préalablement dialysées et lyophylisées (CEP).

La motilité des spermatozoïdes dans les différents échantillons a été examinée après 10 min d'incubation à $34^{\circ} \mathrm{C}$ car l'existence d'un phénomène d'agglutination des spermatozoïdes dans certaines régions épididymaires (Dacheux et al., 1983) rend pratiquement impossible l'analyse de la motilité dans des échantillons incubés au-delà de $15 \mathrm{~min}$.

\section{Analyse de la motilité.}

La motilité des spermatozoïdes a été examinée par quatre techniques subjectives et objectives.

a) Estimation visue/le. - Le pourcentage de spermatozoïdes motiles et progressifs a été estimé de façon subjective au microscope à contraste de phase après l'observation d'une goutte d'échantillon placée entre lame et lamelle.

b) Analyse par effet Doppler. - Le pourcentage des spermatozoïdes a été apprécié de façon objective par la méthode utilisant une source laser et un système de mesure de la variation, par effet Doppler, de la longueur d'onde de la 
lumière diffusée par les spermatozoïdes mobiles (Spermokinésimètre commercialisé par SORO Arcueil $F$ ). Les résultats concernant la validité de cet appareil ont été décrits par Paquignon et al. (1983). La mesure de la motilité dans ces conditions a été effectuée à $34^{\circ} \mathrm{C}$ dans une cellule d'épaisseur de $100 \mu \mathrm{m}$.

c) Analyse par microphotographies. - Des clichés photographiques $(24 \times 36 \mathrm{~mm})$ ont été pris en poses d'une à quatre secondes à partir d'échantillons en fond noir observés entre lame et lamelle. Le pourcentage de cellules mobiles a été estimé par comptage des traces sur les tirages positifs agrandis 10 fois.

d) Analyse microcinématographique. - Des séquences cinématographiques à partir de film Kodak Plus X 50 ASA ont été effectuées en contraste interférentiel avec un objectif de $\times 25$ à une vitesse de prise de vues de 50 ou 64 images par seconde sur un aliquote de $20 \mu \mathrm{l}$ d'échantillon, placé entre lame et lamelle $(20 \times 20 \mathrm{~mm})$ à $34^{\circ} \mathrm{C}$. Trois à six champs choisis au hasard ont été filmés pendant environ quatre secondes de façon à obtenir au minimum cinquante spermatozoïdes par échantillon. Pour l'analyse, le film a été projeté image après image sur une table avec un grossissement final de 1500 .

Les pourcentages de spermatozoïdes mobiles, progressifs (quels que soient les déplacements et les formes de progression) et rotatifs ont été évalués sur l'ensemble des échantillons. Des dessins des positions successives des spermatozoïdes correspondant à une seconde d'enregistrement (51 ou 65 images), ont permis de calculer les paramètres suivants :

- La vitesse de progression $(V p)$ (distance parcourue par la tête des spermatozoïdes dans l'axe de la direction de progression et exprimée en $\mu \mathrm{m} / \mathrm{sec}$ ).

- L'enveloppe des battements flagellaires $(E)$ (largeur maximale de la surface balayée par le flagelle dans l'axe de progression).

- La fréquence de battement flagellaire $(\mathrm{Fb})$ (nombre d'ondes flagellaires formé par seconde sur deux cycles consécutifs).

- La fréquence de rotation des gamètes (nombre de retournement de la tête du gamète par seconde).

\section{Analyse statistique.}

Les résultats ont été comparés par analyse de variance à plusieurs facteurs et par la méthode des contrastes (Snedecor et Cochran, 1971).

\section{Résultats.}

1. Evolution de la motilité des spermatozö̈des au cours de leur transit dans l'épididyme.

Les résultats concernant le pourcentage de spermatozoïdes mobiles et progressifs sont présentés dans le tableau 1. Le pourcentage de motilité des spermatozoïdes a été évalué par différentes techniques et une très bonne 
correspondance a pu être établie entre celles-ci (tabl. 1). Le pourcentage de motilité augmente entre les régions 4 et $9(P<0,01)$ (tabl. 2$)$. En présence de BSA, un important phénomène d'agglutination rend impossible une étude de la motilité dans la plupart des régions épididymaires (résultats non rapportés). En présence de CEP, une augmentation importante du pourcentage de spermatozoïdes motiles est observée dans les quatre régions épididymaires.

\section{TABLEAU 1}

Corrélation entre les différentes techniques d'évaluation du pourcentage de motilité, $R(n)$

\begin{tabular}{lccrr}
\hline & Microcinéma & Obs. Visuelle & Spermokiné. & Microphoto \\
\hline Microcinéma & $1.0(37)$ & $0.84(31)$ & $0.82(31)$ & $0.88(31)$ \\
Obs. Visuelle & & $1(31)$ & $0.90(31)$ & $0.90(25)$ \\
Spermokiné. & & & $1(31)$ & $0.83(25)$ \\
Microphoto & & & & $1(31)$ \\
\hline
\end{tabular}

En milieu salin, des spermatozoïdes progressifs sont observés à partir de la région 5 (tabl. 2) et leur nombre augmente progressivement jusque dans la région terminale de l'épididyme. Toutefois pour une même zone épididymaire, de très fortes variations dans les pourcentages de spermatozoïdes progressifs apparaissent entre animaux. L'addition de protéines au milieu d'incubation a une action positive sur l'évolution de la motilité progressive. Des spermatozoïdes progressifs sont observés dès la région 4 et leur nombre augmente très fortement dans les autres régions.

\section{TABLEAU 2}

Evolution du pourcentage de spermatozoìdes motiles et progressifs en fonction des régions épididymaires et de la présence dans le milieu d'incubation de $5 \mathrm{mg} / \mathrm{m} /$ de protéines épididymaires. (Moyenne \pm écart type)

\begin{tabular}{|c|c|c|c|}
\hline $\begin{array}{l}\text { Protéine } \\
\text { (CEP) }\end{array}$ & $\begin{array}{l}\text { Régions } \\
\text { épid. }\end{array}$ & $\%$ motilité & $\%$ progressif \\
\hline- & $\begin{array}{l}4 \\
5 \\
7 \\
9\end{array}$ & $\begin{array}{l}36 \pm 14 \\
58 \pm 12 \\
59 \pm 18 \\
66 \pm 15\end{array}$ & $\begin{array}{r}0 \pm 0 \\
6 \pm 17 \\
17 \pm 26 \\
17 \pm 33 \\
\end{array}$ \\
\hline+ & $\begin{array}{l}4 \\
5 \\
7 \\
9\end{array}$ & $\begin{array}{l}73 \pm 17 \\
82 \pm 8 \\
88 \pm 10 \\
79 \pm 16\end{array}$ & $\begin{array}{l}55 \pm 35 \\
78 \pm 10 \\
79 \pm 16 \\
70 \pm 15\end{array}$ \\
\hline
\end{tabular}




\section{Analyse du mouvement flagellaire.}

Le mouvement des spermatozoïdes a été analysé par microcinématographie dans toutes les régions épididymaires où des gamètes mobiles ont pu être observés. Les caractéristiques du mouvement des spermatozoïdes sont présentées dans le tableau 3.

TABLEAU 3

Evolution des paramètres quantitatifs ( $\mathrm{Fr}, \mathrm{Fb}$ et $\mathrm{Vp}$ ) en fonction du niveau épididymaire avec et sans CEP. (Moyenne \pm écart type).

\begin{tabular}{lccccc}
\hline Milieu & Niveau & Fr $(\mathrm{Hz})$ & $\mathrm{Fb}(\mathrm{Hz})$ & $\mathrm{E}(\mu \mathrm{m})$ & $\mathrm{Vp}(\mu \mathrm{m} / \mathrm{s})$ \\
\cline { 2 - 6 } & 4 & - & $11,9 \pm 0,9(5)$ & - & - \\
$\mathrm{K}$ & 5 & - & $12,2 \pm 1,1(6)$ & $14,2 \pm 3,0(5)$ & - \\
$\mathrm{R}$ & 7 & - & $14,2 \pm 0,9(10)$ & $14,3 \pm 1,3(8)$ & $48,1 \pm 5,3(9)$ \\
$\mathrm{B}$ & 9 & $5,9 \pm 0,6(17)$ & $19,6 \pm 1,5(12)$ & $10,8 \pm 1,1(13)$ & $83,9 \pm 5,5(21)$ \\
\hline & 4 & - & $10,4 \pm 1,0(10)$ & $15,1 \pm 0,7(9)$ & $32,8 \pm 3,4(13)$ \\
$\mathrm{C}$ & 5 & & $13,4 \pm 0,7(10)$ & $14,5 \pm 0,6(9)$ & $52,1 \pm 5,8(10)$ \\
$\mathrm{E}$ & 7 & $5,0 \pm 0,8(10)$ & $15,5 \pm 1,5(6)$ & $15,3 \pm 0,9(6)$ & $73,8 \pm 4,6(21)$ \\
$\mathrm{P}$ & 9 & $6,1 \pm 0,7(17)$ & $18,8 \pm 1,9(11)$ & $11,4 \pm 0,9(12)$ & $83,5 \pm \mathbf{4 , 2}(23)$ \\
\hline
\end{tabular}

Abréviations : vơir Matériel et Techniques, $2 \mathrm{~d}$.

La fréquence des battements flagellaires augmente significativement $(P<0,01)$ entre les zones 4 et 9 et les valeurs sont sensiblement les mêmes en présence ou non de protéines dans le milieu d'incubation. En milieu salin, la vitesse de progression double entre les régions 7 et 9 . En milieu protéique, l'apparition de spermatozoïdes progressifs est déplacée vers les régions antérieures de l'épididyme et leur vitesse de progression augmente significativement au cours du transit $(P<0,01)$. La rotation des gamètes qui apparaît dans la région 9 en milieu salin est observée dès la région 7 en présence de CEP.

La forme de la trajectoire des gamètes mobiles évolue avec la maturation. La figure 1 illustre les mouvements les plus significatifs des spermatozoïdes prélevés à différents niveaux de maturation. Dans la zone 4, en milieu salin, les spermatozoïdes présentent seulement des oscillations régulières du flagelle mais d'efficacité nulle (aucun déplacement significatif). En présence de protéines dans le milieu d'incubation, le battement flagellaire devient efficace et induit un mouvement circulaire de très faible rayon centré sur la tête du spermatozoïde. L'onde flagellaire (zone de courbure) englobe pratiquement la totalité du flagelle (fig. 1). Dans la zone 5, plusieurs courbures successives apparaissent sur le flagelle dans des directions opposées. Une asymétrie existe dans le développement de ces ondes: I'une (onde principale) est très développée, l'autre (onde reverse) est réduite. Le déplacement des gamètes induit par ce type de battement est toujours circulaire mais de rayon beaucoup plus grand que dans la zone précédente. Dans la zone 7, l'asymétrie dans le développement des courbures 


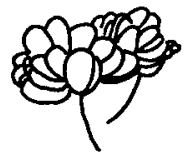

4
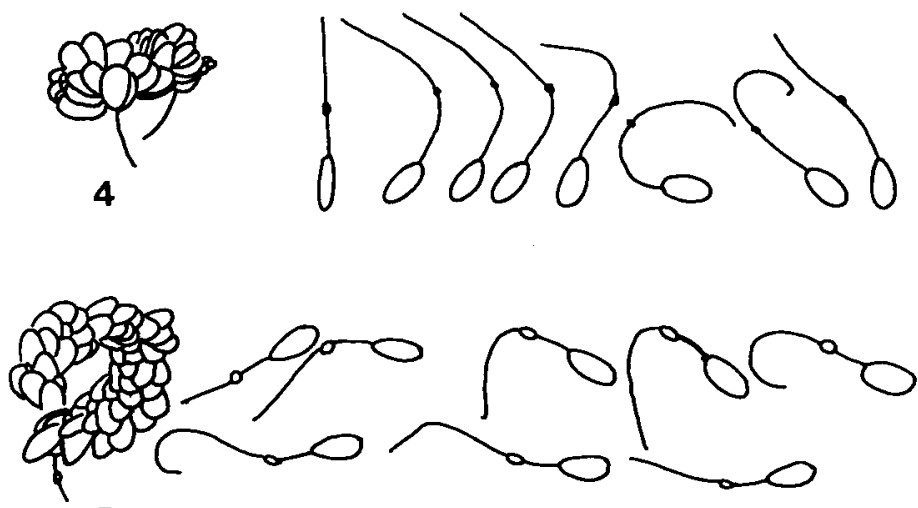

5

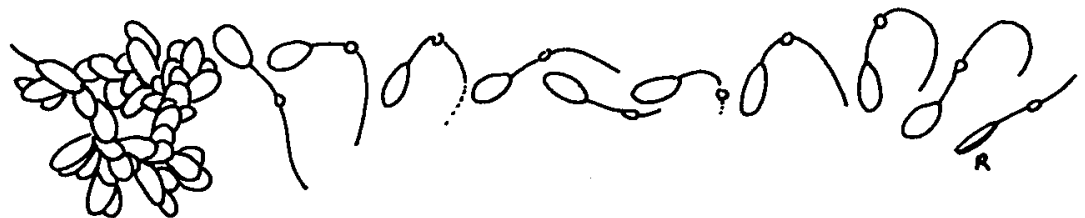

7

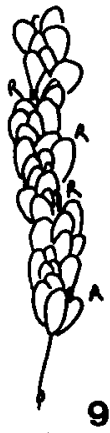

9

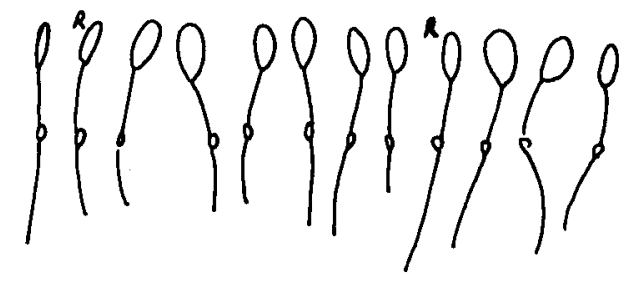

$50 \mathrm{um}$

FIG. 1. - Tracés des positions successives de la tête des spermatozö̈des de verrat et des formes flagellaires caractéristiques correspondantes durant une seconde d'enregistrement cinématographique selon les zones de prélèvements dans l'épididyme (4 à 9). R : Rotations.

flagellaires diminue. Une composante linéaire apparaît dans le déplacement des gamètes rendant la trajectoire alternativement rectiligne et circulaire. Alors que dans la région 4 de la majorité du battement flagellaire s'effectue dans un plan, à partir de la région 7 une composante tridimensionnelle apparaît. Elle est visible principalement dans la partie terminale du flagelle et provoque sa sortie du plan 
focal de l'enregistrement cinématographique. Cette nouvelle composante du battement flagellaire induit une rotation des gamètes dans la région 9 en milieu salin et, dès la région 7 après addition de CEP. La fréquence de rotation qui est toujours inférieure à la fréquence de battement du flagelle est d'environ $6 \mathrm{hz}$ pour les spermatozoïdes prélevés dans la région caudale de l'épididyme, ceci en présence ou en absence de protéines dans le milieu d'incubation. Dans la zone 9 , l'amplitude des courbures flagellaires est plus faible que dans les zones précédentes et un déplacement rectiligne des spermatozoïdes est obtenu grâce aux rotations successives. Quel que soit le milieu d'incubation utilisé, dans la région caudale existe une grande variabilité entre animaux concernant le pourcentage de gamètes progressifs.

\section{Discussion.}

L'évolution du mouvement des spermatozoïdes au cours de leur transit dans l'épididyme a été établie chez le verrat à partir de quatre zones épididymaires dans lesquelles des modifications importantes dans la composition des protéines de surface membranaire des gamètes ont été décrites (Dacheux et al., 1985). Ces principaux changements de surface sont représentés par la disparition ou le masquage dans la région antérieure (région 4 comprise) de la majorité des protéines de surface mises en évidence sur les gamètes testiculaires, par la présence dans la région médiane (région 5) d'un état transitoire de la surface membranaire et par l'apparition dans la région 7 de nouveaux composés protéiques qui caractérisent le revêtement du spermatozoïde mature de la région caudale (région 9) (Dacheux et al., 1985).

L'étude de la motilité des spermatozoïdes concerne aussi bien l'évaluation d'une population hétérogène de gamètes animés de mouvements plus ou moins coordonnés que la description même du battement flagellaire à l'origine de ces mouvements. Ces différents paramètres sont actuellement difficilement accessibles avec une seule technique d'analyse.

L'évolution des pourcentages de spermatozoïdes motiles et progressifs obtenue en milieu salin correspond à celle obtenue précédemment sur cette même espèce (Paquignon et al., 1983) (Jeulin et al., 1987). La présence de protéines (CEP) dans le milieu d'incubation modifie ce gradient, en augmentant le pourcentage de spermatozoïdes mobiles dans toutes les zones de l'épididyme. Par contre, elle ne modifie pas les caractéristiques fondamentales du battement flagellaire : fréquence de battement et degré d'angulation. Cet effet sur la mobilité totale est très certainement lié à une action antiadhésive de ces composés vis-à-vis de la membrane des spermatozoïdes avec la surface du verre des lames et lamelles (Stephens et al., 1981). Cependant cette interaction des gamètes avec la surface des lames varie selon la puissance développée dans le battement flagellaire: une cellule peu mobile aura tendance à adhérer à la surface tandis qu'une cellule très progressive pourra plus facilement s'en détacher. Une action antiadhésive de la BSA existe également mais celle-ci n'a pu être utilisée dans cette étude car sa présence accélère l'apparition de l'agglutination. 
La maturation du mouvement flagellaire se traduit par une élévation de la fréquence du battement lors du transit des gamètes dans l'épididyme. Ce résultat implique une augmentation de la vitesse de propagation de l'onde flagellaire, une diminution de sa longueur ainsi que la réduction de la courbure du flagelle. De telles modifications ont été également observées chez le bélier (Chevrier et Dacheux, 1986, 1988). L'existence d'une succession régulière d'ondes sur le flagelle provoque l'apparition d'un déplacement du gamète et la réduction progressive de l'inégalité des courbures entre les deux côtés du flagelle produit un mouvement de moins en moins circulaire. Le déplacement rectiligne chez le verrat n'apparaît que lorsque des rotations du gamète ont lieu. Ces rotations induites par une propagation dans les trois dimensions de l'onde flagellaire caractérisent le mouvement flagellaire mature du spermatozoìde. A de rares exceptions, quelles que soient les conditions d'incubation, ce mouvement n'apparaît que dans les régions caudales de l'épididyme. L'augmentation de la vitesse de déplacement des spermatozoïdes $V p$ durant la maturation, s'accompagne d'une augmentation de l'efficacité du battement $(\mathrm{Vp} / \mathrm{Fb})$ qui passe de 3 à 4,2 entre le début et la fin de l'épididyme. L'efficacité flagellaire calculée chez le verrat est du même ordre de grandeur que celle obtenue chez l'Homme (3,9: Serres et al., 1984), le Bélier (4,7: Denehy, 1975) ou le Taureau (4,4: Rikmenspoel, 1965). La présence de protéines dans le milieu d'incubation ne change pas ces paramètres.

La mise en place du mouvement flagellaire dans le spermatozoïde épididymaire de verrat coïncide avec les grands changements membranaires à la surface des gamètes. Cependant aucune relation entre les composés de surface et une activité flagellaire n'a pu être mise en évidence. L'absence d'effet notable des protéines épididymaires de la région caudale sur le mouvement flagellaire de type immature suggère une faible relation entre ce milieu protéique et une induction du mouvement. La séquence des modifications que subissent les spermatozoïdes au cours du transit épididymaire implique qu'une très forte interaction existe entre l'évolution du gamète et les modifications de son environnement, mais les signaux permettant de modifier le fonctionnement très régionalisé du système dynéinetubuline de l'axonème sont inconnus.

$5^{e}$ Congrès de la Société d'Andrologie de langue française, Paris, décembre 1987.

Remerciements. - Travaux subsidiés par les contrats de recherche INSERM n ${ }^{\circ} 135007$ et 854006 .

\section{Références}

BEDFORD J. M., 1975. Maturation, transport and fate of spermatozoa in the epididymis, 303-317. In HAMILTON D.W., GREEP R. O., Handbook of Physiology, sect. 7 ; Endocrinology, vol. V. Am. Physiol. Soc., Washington D.C.

CHEVRIER C., DACHEUX J. L., 1986. Motility index and flagellar wave parameters in mammalian sperm. Cell Motil. Cytoskel., 6, p. 207.

CHEVRIER C., DACHEUX J. L., 1988. Analysis of the flagellar bending waves during the maturation of ram sperm in the epididymis. Cell Motility and the Cytoske/eton (in press). 
DACHEUX J. L., PAQUIGNON M., COMBARNOUS Y., 1983. Head-to-head agglutination of ram and boar epididymal spermatozoa and evidence for an epididymal antagglutinin. J. Reprod. Fert., 67, 181-189.

DACHEUX J. L., PAQUIGNON M., LANNEAU M., 1985. Sequential analysis of the epididymal sperm maturation process in the boar, 526-529. In CATT K. J., DUFAU M. L., Hormone action and testicular function. New York Acad. Sci.

DENEHY M. A., 1975. The propulsion of non rotating ram and oyster spermatozoa. Biol. Reprod., 13, 17-29.

GADDUM P., 1968. Sperm maturation in the male reproductive tract : development of motility. Anat. Rec., 161, $471-482$.

IMAI H., NIWA K., IRITANI A., 1977. Penetration in vitro of zona free hamster eggs by ejaculated spermatozoa. J. Reprod. Fert., 51, 495-497.

JEULIN C., SOUFIR J. C., MARSON J., PAQUIGNON M., DACHEUX J. L., 1987. The distribution of carnitine and acetylcarnitine in the epididymis and epididymal spermatozoa of the boar. J. Reprod. Fert., 79, 523-529.

ORGEBIN-CRIST M. C., DANZO B. J., DAVIES J., 1975. Endocrine control of the development and maintenance of sperm fertilizing ability in the epididymis, 319-338. In HAMILTON D. W., GREEP R. O., Handbook of physiology, sect. 7, vol. V. Am. Physiol. Soc., Washington D.C.

PAQUIGNON M., DACHEUX J. L., JEULIN C., FAUQUENOT A., 1983. Laser light scattering study of spermatozoa motility of domestic animals, 332-335. In ANDRE J., The sperm cell, N. Nijhoff, The Hague.

RIKMENSPOEL R., 1965. The tail movement of bull spermatozoa. Observations and model calculations. Biophys. J., 5, 365-392.

SERRES C., FENEUX D., JOUANNET P., DAVID G., 1984. Influence of the flagellar wave development and propagation on the human sperm movement in seminal plasma. Gam. Res., 9, 183-195.

SNEDECOR G. W., COCKRAN W. G., 1971. Statistical methods. The lowa State Univ. Press, Ames. STEPHENS D. T., ACOTT T. S., HOSKINS D. D., 1981. A cautionary note on the determination of FMP : Activity with bovine epididymal spermatozoa. Biol. Reprod., 25, 945-949. 